\title{
Emotion Dysregulation and Internalizing Spectrum Disorders
}

The Oxford Handbook of Emotion Dysregulation

Camelia E. Hostinar ${ }^{1}$, Ph.D. \& Dante Cicchetti ${ }^{2}$, Ph.D.

1. University of California -Davis, Psychology Department

2. University of Minnesota, Institute of Child Development

Please cite this chapter as:

Hostinar, C. E., \& Cicchetti, D. (2020). Emotion dysregulation and internalizing

spectrum disorders. In T. P. Beauchaine \& S. E. Crowell (Eds.), Oxford Handbook of

Emotion Dysregulation. doi: 10.1093/oxfordhb/9780190689285.013.18

This paper is the authors' copy and may not exactly replicate the final version of the article in the Oxford Handbook of Emotion Dysregulation.

Acknowledgements: Authors effort on this manuscript was supported by funding from the NSF (SMA1327768) to Camelia E. Hostinar and from the Jacobs Foundation to Dante Cicchetti.

Correspondence regarding this manuscript should be addressed to Camelia E. Hostinar, Ph.D. (cehostinar@ucdavis.edu) or Dante Cicchetti, Ph.D. (cicchett@umn.edu). 


\begin{abstract}
Emotion dysregulation is a pattern of emotional experience or expression that interferes with goal-directed behavior, and may set children and adolescents on developmental trajectories towards psychopathology. Despite accumulating evidence linking emotion dysregulation to various forms of internalizing psychopathology (e.g., depression, anxiety), several open questions remain. What are the developmental pathways leading to emotion dysregulation and modifiable environmental conditions that appear to foster it? What is the evidence that emotion dysregulation precedes or plays a causal role in the development of internalizing spectrum disorders? What are the biological underpinnings of emotion dysregulation and how can we integrate this research with recent discoveries about internalizing spectrum disorders from neuroscience, psychophysiology, genetics, and epigenetics? This review discusses recent research pertinent to these three primary questions and concludes with suggestions for future research and implications for interventions that promote resilience in youth.
\end{abstract}

Keywords: emotion, emotion dysregulation, child, adolescent, psychopathology, internalizing 


\section{Emotion Dysregulation and Internalizing Spectrum Disorders}

Our ability to manage difficult negative emotions is central for our mental and physical health, successful social interactions, and for accomplishing long-term goals. In contrast, emotion dysregulation is a pattern of emotional experience or expression that interferes with goal-directed behavior, and may set us on trajectories towards psychopathology (Beauchaine \& Gatzke-Kopp, 2012). This failure to modulate emotional dynamics to achieve beneficial outcomes can occur for any number of reasons. It can arise from the intensity of the emotions (which can make them more challenging to regulate), from mismatches between the emotional experience and the situation, or from problems of coordination between emotion and cognition, or between emotion and action (Cicchetti, Ackerman, \& Izard, 1995). Understanding the diverse causes and consequences of emotion dysregulation is critical because it is a defining feature of many debilitating forms of psychopathology, particularly internalizing disorders like depression and anxiety (Aldao, Nolen-Hoeksema, \& Schweizer, 2010; Behar, DiMarco, Hekler, Mohlman, \& Staples, 2009; Gotlib \& Joormann, 2010; Mennin, Heimberg, Turk, \& Fresco, 2002; Sheppes, Suri, \& Gross, 2015).

However, despite the heuristic value and accumulating empirical evidence supporting the utility of this construct for understanding the development and maintenance of psychopathology in general and internalizing spectrum disorders in particular (Beauchaine, 2015a; Cole \& Hall, 2010), many open questions remain. For instance, what are the developmental pathways leading to emotion dysregulation and modifiable environmental conditions that appear to foster it? What is the evidence that emotion dysregulation precedes or plays a causal role in the development of internalizing spectrum disorders, as opposed to simply being a correlate or consequence of psychopathology? Furthermore, what are the biological underpinnings of emotion dysregulation 
and how can we integrate this research with recent discoveries about internalizing disorders from neuroscience, psychophysiology, genetics, and epigenetics? This review aims to discuss research focusing on these questions and has three goals. First, we summarize leading theoretical perspectives on the developmental origins of and potential pathways to emotion dysregulation. Second, we review current methods of assessing emotion regulation and dysregulation in children and adolescents, and highlight some of the most recent findings supporting its role as a specific and potentially causal risk factor that precedes the development of internalizing spectrum disorders. Third, we describe recent research attempting to link emotion regulation/dysregulation with multilevel processes at neural, psychophysiological, genetic, and epigenetic levels of analysis. Finally, we conclude by providing some future directions for research and implications for interventions and the promotion of resilience. We adopt a developmental psychopathology framework (Cicchetti, 1984, 2016) throughout this review because we believe that understanding normal and abnormal trajectories of emotional development across childhood and adolescence can yield important insights into adult endpoints like severe emotion dysregulation and clinical levels of symptomatology. Furthermore, this developmental approach can inform decisions on when, where, and how to intervene before maladaptive emotional patterns become fully consolidated. Before proceeding with these central aims, we define the primary concepts of interest in this review.

\section{Defining the Core Concepts}

Despite lingering theoretical disagreements on the ultimate nature of emotions, many scientists agree that emotions are processes that motivate and organize behavior (Cicchetti et al., 1995), and they reflect an appraisal of a situation's meaning for individual wellbeing (Cole, Martin, \& Dennis, 2004). An important realization in research from the past two decades has 
been that the qualities of our emotional experiences (e.g., valence, intensity, duration) depend on our ability to modify emotional responses to accomplish individual goals, which has been termed emotion regulation (Thompson, Lewis, \& Calkins, 2008). The challenge of measuring emotion and its regulation as separate processes has raised a number of questions about the validity of the construct of emotion regulation (Cole et al., 2004). However, two streams of evidence have provided foundational evidence for the validity and utility of the construct. First, experimental paradigms capturing independent manifestations of emotion and emotion regulation demonstrate that these processes can be studied separately despite being closely related (Cole et al., 2004) e.g., in infants, facial expressions of emotion and behavioral indicators of emotion-regulatory strategies such as gaze aversion and self-soothing can be captured independently from each other). Secondly, studies identifying different neural correlates for various emotion regulation strategies such as cognitive reappraisal and emotion suppression elicited in the laboratory (e.g., Goldin, McRae, Ramel, \& Gross, 2008) suggest the utility of describing and classifying various forms of emotion regulation to allow for a closer mapping of complex thoughts and behaviors onto their corresponding neural circuitry.

Another compelling rationale for studying emotion regulation is to understand why and when it goes awry, a phenomenon frequently observed in psychopathology. As mentioned, emotion dysregulation can be defined as a pattern of emotional experience or expression that interferes with goal-directed behavior (Beauchaine \& Gatzke-Kopp, 2012). Leading theories on the development of many types of psychopathology assign important etiological roles to emotion dysregulation (Aldao et al., 2010; Behar et al., 2009; Gotlib \& Joormann, 2010; Mennin et al., 2002; Sheppes et al., 2015). These theories also specify different types of emotion-regulatory failures as being involved in different disorders - e.g., the inability to disengage from negative 
affect is more characteristic of depression (Gotlib \& Joormann, 2010), whereas overuse of avoidance and suppression of emotion are thought to promote chronic worry and anxiety disorders (Mennin et al., 2002). While these theories have garnered significant empirical support in the last two decades, an ongoing challenge in this area of research is that psychopathology can be a result of problems with emotion generation, emotion regulation, or both. Thus, it can be difficult to isolate the specific effects of emotion regulation difficulties in the development of psychopathology (Cicchetti et al., 1995; Sheppes et al., 2015). However, as we discuss below, there is emerging evidence to suggest a unique and additive role of emotion dysregulation in explaining variability in mental health outcomes above the role of other constructs such as trait negative affectivity or exposure to stressful life events.

In this review we focus specifically on internalizing spectrum disorders, which are psychological conditions with high levels of negative affectivity that is self-directed or “intropunitive" and is most often covert (Tandon, Cardeli, \& Luby, 2009). In the DSM-5 classification system, these include depressive disorders, anxiety disorders, obsessivecompulsive and related disorders, trauma and stressor-related disorders, and dissociative disorders (Regier, Kuhl, \& Kupfer, 2013). In this review, we focus primarily on depressive and anxiety disorders, as most prior research on emotion dysregulation with children and adolescents has centered on these subtypes of internalizing disorders. Internalizing spectrum disorders are often characterized by ineffective management of emotional arousal (particularly sadness or fear), and are typically accompanied by alterations in physiology and neural function. These disorders are also more prevalent among those who have experienced major life stressors or emotionally unsupportive environments during childhood or adolescence, as we discuss in the next section. Unlike temperamental predispositions for internalizing symptoms, which show 
moderate levels of heritability, emotion dysregulation is one of the less heritable risk factors for psychopathology (Beauchaine, 2015a). This suggests an important role for the social environment in shaping children's ability to develop consistent and effective strategies to regulate their emotions, and the greater potential for plasticity if interventions occur early in development.

\section{The Developmental Origins of Emotion Dysregulation}

The social milieu plays an important role in fostering child and adolescent emotion dysregulation and subsequent psychopathology. Childhood maltreatment, insecure or disorganized attachment, parental psychopathology, negative family climate and practices, and peer rejection and victimization have all been linked to greater odds of emotion dysregulation and internalizing psychopathology. What remains to be understood are the pathways through which rejecting or unpredictable family and peer groups lead to these outcomes, though some evidence is beginning to illuminate these pathways.

Family processes. Maltreatment is a toxic social environment for children that poses significant risk for the development of both internalizing and externalizing psychopathology (Cicchetti \& Ng, 2014; Cicchetti \& Toth, 2005). For instance, recent meta-analytic syntheses reveal that childhood maltreatment experiences are associated with greater risk of developing depression and anxiety (Li, D'Arcy, \& Meng, 2016; Nanni, Uher, \& Danese, 2012; Nelson, Klumparendt, Doebler, \& Ehring, 2017). Furthermore, maltreatment predicts an earlier onset, a more severe course, and greater risk of recurring, persistent, and treatment-resistant manifestations of mood disorders (Nanni et al., 2012; Nelson et al., 2017). Despite these robust associations, there is a dearth of studies examining the protective factors or other modifiable 
predictors of depression following maltreatment (Braithwaite, O'Connor, Degli-Esposti, Luke, \& Bowes, 2017).

Nevertheless, research from the past two decades has consistently revealed that maltreated children have emotional difficulties with emotion perception, understanding, and regulation (Cicchetti \& Ng, 2014; Luke \& Banerjee, 2013; Pollak, 2015), and these alterations likely explain a number of pathways to mood and anxiety disorders. For instance, neglected children struggle with discriminating emotional expressions, whereas physically abused children show attentional biases towards angry faces (Pollak, Cicchetti, Hornung, \& Reed, 2000). Maltreated children also show greater negative affect during tasks that provoke anger or frustration (Shackman \& Pollak, 2014). More specifically relevant to the development of depression, maltreated children show biased attention for sad faces after the induction of a sad state, particularly if they have an affective style that is high in rumination, an emotion-regulation strategy that is ineffective in dampening negative affect and that is a risk factor for depression (Romens \& Pollak, 2012). A recent meta-analysis of 51 empirical studies showed that of all the emotional deficits studied in maltreated children, the greatest impairments are in the domain of emotion understanding and these impairments are most prevalent in younger children compared to older children (Luke \& Banerjee, 2013).

One of the primary pathways through which maltreatment leads to emotion dysregulation appears to be through altered attentional processes, which can involve either poor attention modulation and attention deficits (Shields \& Cicchetti, 1998) or attentional biases towards threatening or sad stimuli (for a recent review, please see Pollak, 2015). These factors can lead children to dedicate more attentional resources to negative stimuli, which generates more negative affect that is then compounded by difficulties with disengaging or shifting attention 
away from negative stimuli. In addition to these biases, there is some evidence that another important pathway may be that maltreated children develop atypical patterns of processing positive stimuli and rewards. For instance, one study with 8-14-year-olds revealed that maltreated youth were less likely to modulate their behavioral responses based on rewards, and those maltreated youth with depressive disorders were more likely to prefer low risk/low reward options than high risk/high reward options (Guyer et al., 2006). It is unclear whether this is due to the relative infrequency of rewards in the environment for maltreated children, or due to stress-related neural alterations in reward circuitry that shifts how rewards are processed. Overall, attention control deficits, attentional biases to threat, and blunted responsivity to rewards form a triad of characteristics that may not only independently increase the odds of developing depressive symptomatology, but may potentiate each other over time, though this possibility has yet to be empirically tested.

While these extreme forms of harsh or neglectful parenting can predispose children to emotion dysregulation and subsequent psychopathology, other family characteristics and practices can have similar effects, even in the absence of full-blown abuse or neglect. There are three primary pathways through which families are thought to impact the development of children's emotion regulation. First, children first learn how to regulate their emotions from their families via observation, which includes processes like observational learning, modeling, and social referencing (Morris, Silk, Steinberg, Myers, \& Robinson, 2007; Thompson \& Meyer, 2007). Secondly, there is also evidence that specific parenting practices and behaviors socialize children about emotions and their regulation (Calkins \& Hill, 2007). For instance, parents' negative reactions to children's emotions as well as parents' own limitations in emotional expressivity can set children on trajectories towards emotion dysregulation (Calkins \& Hill, 
2007; Eisenberg, Cumberland, \& Spinrad, 1998). Finally, the emotional climate of the family, which includes the overall relationship quality and the attachment processes embedded in the parent-child relationship and marital relationship, also shape children's emotion regulation abilities for better or for worse (Brumariu \& Kerns, 2010; Morris et al., 2007). Attachment research has shown that sensitive, responsive caregiving teaches children to use their parental figures as a safe haven and an external regulator of distress and other negative emotions (Ainsworth, Bell, \& Stayton, 1974). Over time, these processes become internalized and children begin to be able to regulate their own negative emotions. However, in situations of inconsistent, emotionally unavailable, or frightened/frightening parenting, children develop insecure or disorganized attachment patterns and emotion regulation strategies that are not effective in downregulating negative affect (Main \& Solomon, 1986). Children who develop an insecureanxious attachment pattern learn to amplify their own negative and fearful behaviors to elicit caregiving from inconsistent parents, whereas those with an insecure-avoidant pattern learn to suppress and mask their negative affect to avoid eliciting rejection from their caregivers (Cassidy, 1994). Moreover, children with disorganized attachment strategies develop incoherent and contradictory strategies that leave them vulnerable in the face of environmental challenges (Brumariu \& Kerns, 2010). While all of these strategies are well adapted to the specific caregiving circumstances children find themselves in, they are not effective at managing negative affect in the long-run and may predispose to psychopathology. Indeed, a recent review of this empirical literature showed that overall most studies suggest that early-life insecure attachment is related to a higher chance of subsequently developing depression or anxiety, with associations being stronger when psychopathology is assessed in preadolescence and adolescence versus childhood (Brumariu \& Kerns, 2010). However, few studies have directly 
tested the hypothesis that emotion dysregulation mediates these association. One report from the Minnesota Longitudinal Study of Parents and Children supported this hypothesis and revealed that a history of attachment insecurity assessed with the Strange Situation procedure during infancy was associated with parent- and teacher-reported anxiety in childhood, and this association was mediated by preschool emotion regulation skills as assessed by levels of distress, crying, and anger during a frustration task (Bosquet \& Egeland, 2006). Another study examining attachment representations in middle childhood and adolescence (ages 8-14 years old) found that attachment anxiety was related to emotion dysregulation, whereas attachment avoidance was associated with emotion suppression, and emotion dysregulation and suppression partially mediated associations with depressive symptoms cross-sectional mediation analysis (Brenning, Soenens, Braet, \& Bosmans, 2012).

Children's biological predispositions also interact with their environments to shape their risk of developing internalizing spectrum disorders. For instance, children whose temperaments are high on behavioral inhibition or negative affectivity tend to develop more symptoms of anxiety or depression when reared in negative, unsupportive environments (Dougherty, Klein, Rose, \& Laptook, 2011; N. A. Fox, 1994; Yap, Allen, \& Sheeber, 2007). Parental psychopathology is another risk factor for children's internalizing symptoms, whether through genetic risk factors or through dysfunctional patterns of parenting - e.g., parents with borderline personality disorder tend to engage in more insensitive, hostile, or overprotective parentings, which in turn predicts children's psychopathology (Eyden, Winsper, Wolke, Broome, \& MacCallum, 2016). Moreover, these parents may also possess biological vulnerabilities that serve as a diathesis for emotional lability in their offspring. 
Peer processes. In addition to the family environment, relationships with peers can also promote or hinder the development of emotion regulation abilities, as well as subsequent psychopathology. Children between the ages of 8-13 years old who are victims of relational or overt victimization are at greater risk of developing internalizing symptomatology, though this association is moderated by genotype -e.g., the serotonin transporter gene variant (Banny, Cicchetti, Rogosch, Oshri, \& Crick, 2013). Longitudinal studies show the complex interplay between family and peer relationships, emotion dysregulation, and psychopathology. For instance, one such study revealed that children aged 6-12 years old who had experienced maltreatment by family members were more likely to develop emotion dysregulation, which was linked to higher externalizing psychopathology and later peer rejection (Kim \& Cicchetti, 2010). In turn, peer rejection was related to higher levels of psychopathology, whereas higher levels of emotion regulation served as a protective factor against escalating levels of internalizing symptoms over time (Kim \& Cicchetti, 2010). Another large longitudinal study of 1,065 adolescents between the ages of 11 and 14 years old showed that peer victimization predicted increases in emotion dysregulation over a 4-month period, which mediated increases in internalizing symptoms over a 7-month period (McLaughlin, Hatzenbuehler, \& Hilt, 2009). Given the frequency and salience of peer-related stressors in middle school and high school, these findings have important implications for understanding potentially modifiable risk factors for the development of emotion dysregulation and subsequent internalizing symptoms in these age groups.

\section{Current Methods and Recent Findings Explicating the Role of Emotion Dysregulation} in Youth Psychopathology 
Current methods. Emotional processes encompass behavioral, physiological, phenomenological, cognitive, and sociocultural aspects (Solomon, 2002) that are challenging to capture through any single measure or protocol. Thus, it is not surprising that there is a diversity of approaches to assessing emotions and to inferring emotion-regulatory processes. Four general strategies are widely used in research with children and adolescents: self-report, other-report (e.g., from teachers, parents, peers), observation, and neurophysiology (Zeman, Klimes-Dougan, Cassano, \& Adrian, 2007). Additionally, the assessment tools need to be tailored to the developmental stage of the subject. For instance, observation and neurophysiological assessments (e.g., EEG, EKG, hormone production) would be primary sources of information in infancy, whereas self-report becomes more central during childhood and adolescence, but would need to be combined with other measures to enhance validity. In addition to capturing developmental stage, another temporal dimension that would need to be considered is whether the measures reflect state or trait-level processes.

Even within each of these four general approaches, there is a wide diversity of assessment methods. For instance, a recent review of studies from the past 35 years conducted with samples ranging from infancy to adolescence revealed that these studies have used 28 selfreport formats, 17 other-report formats, 47 observational paradigms, and 8 physiological assessments to measure emotion regulation (Adrian, Zeman, \& Veits, 2011). Given this diversity of approaches, there is a critical need for increased standardization of methods and for holistic models that could integrate information from these multimethod and multilevel approaches into a coherent representation of emotional processes that will translate well to real-life and clinical settings. In addition, the models developed should be informative in predicting behavior. 
Another limitation of existing methods is that so far most assessments of emotions and their regulation/dysregulation have focused on the individual, despite the fact that most emotion theories recognize the person-environment interactions inherently involved in any emotional responses. The social-contextual embedding of individuals within their environments is not usually considered in the measurement of emotions, and this is a limitation of current research (Aldao, 2013). For instance, research thus far has not dedicated sufficient attention to questions such as: how typical are the observed emotional reactions for the social, economic, and cultural background of the participants? How do individual emotion regulation skills vary across social contexts and how can we intervene to prevent the generalization of emotion dysregulation from one context (e.g., maltreating family) to others (e.g., school settings and interactions with peers)? Currently, there are no empirically validated taxonomies for capturing the social contexts of emotion (Zeman et al., 2007), thus this remains an important direction for future research.

Recent findings. Numerous correlational studies have now substantiated the hypothesis that emotion regulation strategies are associated with internalizing symptomatology. To illustrate the growing attention to these associations, a recent meta-analysis of 35 empirical studies conducted with youth and aggregating 68 effect sizes confirmed that the habitual use of adaptive emotion regulation strategies (cognitive reappraisal, problem solving, and acceptance) appears protective against depressive and anxious symptoms in adolescence (Schafer, Naumann, Holmes, Tuschen-Caffier, \& Samson, 2017). Cognitive reappraisal involves changing thoughts and beliefs about the meaning of a situation, and is a strategy that alters the emotional process early on before full-blown activation (Gross, 2013). Problem solving is a regulatory strategy that includes cognitive and behavioral responses that attempt to eliminate the cause of negative emotions. Finally, acceptance refers to allowing one's emotions to unfold without judgment or 
suppression (Schafer et al., 2017). In contrast, maladaptive emotion regulation strategies

(avoidance, suppression, and rumination) are positively associated with internalizing symptoms (Schafer et al., 2017). Avoidance consists of averting situations that may trigger negative emotions, whereas suppression refers to attempts to deny or prevent focusing on such emotions, and these strategies have been linked to greater risk of psychopathology in youth (Schafer et al., 2017). Even though avoidance and suppression are associated with worse mental health, the opposite strategy of prolonged mental rehearsal of emotional experiences, their causes and consequences (i.e., rumination) is also linked to greater likelihood of anxiety and depression (Nolen-Hoeksema, Wisco, \& Lyubomirsky, 2008).

However, these overall associations between emotion regulation strategies and internalizing psychopathology do not reveal whether emotion dysregulation precedes or plays a causal role in (versus being a correlate or consequence of) psychopathology. In adults, there is some correlational evidence that emotion regulation explains risk of internalizing psychopathology independently from the explanatory contributions of high-intensity negative affect and of childhood trauma (Bradley et al., 2011), suggesting that the association is not easily explained by other potentially confounding factors. Causal inferences are challenging to extract from correlational data, but prospective longitudinal studies can shed light on the plausible direction of effects and potentially rule out some reverse-causality pathways.

Indeed, one prospective longitudinal study with children (Kim-Spoon, Cicchetti, \& Rogosch, 2013) reported that early-life maltreatment was associated with emotional negativity/lability at age 7 , which was associated with poor emotion regulation at age 8 . This, in turn, predicted internalizing psychopathology at ages 8-9 years old, controlling for prior levels of symptomatology and emotion regulation (Kim-Spoon et al., 2013). This temporal ordering is 
consistent with results from another longitudinal study showing that maternal depression during the first few years of life predicted children's emotion dysregulation at age 3 and worse socioemotional functioning at age 5 (Maughan, Cicchetti, Toth, \& Rogosch, 2007). It is also consistent with a mediational study with 4-6-year-old children revealing that maltreatment history predicted emotion dysregulation, which mediated associations between maltreatment and anxious or depressed symptomatology (Maughan \& Cicchetti, 2002). Importantly, some studies show that parenting moderates the association between emotion dysregulation and subsequent psychopathology. For instance, one study of 9-10-year-old girls showed that low levels of positive affect expression and sadness regulation predict later depressive symptoms only in girls whose parents are low in acceptance (Feng et al., 2009).

Prospective longitudinal studies with adolescents also reveal that emotion dysregulation or poor emotion identification skills can predict increases in symptoms of anxiety, depression, aggression, or eating disorders after controlling for baseline symptoms (Ciarrochi, Heaven, \& Supavadeeprasit, 2008; Hatzenbuehler, McLaughlin, \& Nolen-Hoeksema, 2008; McLaughlin, Hatzenbuehler, Mennin, \& Nolen-Hoeksema, 2011). For instance, one longitudinal study following 667 high school students for one year found that low emotion identification skills in eighth grade predicted increases in fear and decreases in positive affect in the overall sample and increases in sad affect among boys from eighth grade to ninth grade (Ciarrochi et al., 2008). In a community sample of 1,071 middle school students (ages 11-14), sexual minority adolescents reported higher levels of internalizing symptomatology at the baseline assessment and 7 months later (compared to non-minority youth), with emotion regulation deficits mediating changes in internalizing symptoms from baseline to follow-up (Hatzenbuehler et al., 2008). In another report, baseline psychopathology did not predict changes in emotion dysregulation over time but 
baseline emotion dysregulation predicted longitudinal increases in anxiety symptoms, aggressive behavior, and eating pathology among 1,065 middle school students aged 11-14 (McLaughlin et al., 2011).

Overall, these prospective longitudinal studies suggest that emotion dysregulation most consistently precedes the onset or exacerbation of future internalizing symptoms, but the reverse prediction is not true. Causal inference is difficult to draw from correlational findings, but the pattern of results thus far is more consistent with potential causal involvement of emotion dysregulation in the future development of psychopathology, rather than being a consequence of or a simple correlate of psychopathology.

\section{What are the Biological Underpinnings of Emotion Dysregulation? Recent Findings and Open Questions}

Modern conceptualizations of mental health and illness such as the Research Domain Criteria (RDoC) defined by NIMH are moving beyond descriptive, DSM-based categorical definitions of disorders towards understanding the continuum of emotional experiences and pathophysiology (Insel et al., 2010). The goal of this new framework for psychiatric research is to map complex behaviors and symptoms onto their corresponding neural circuits and underlying genetic profiles (Insel et al., 2010). This new generation of research faces the daunting task of integrating multilevel, multimethod measures into coherent models of specific clusters of symptoms that are associated with clinical impairment. Emotion dysregulation could serve as a useful bridge between these multiple levels of analysis, as emotion regulation and dysregulation are thought to be complex psychobiological processes involving multiple neural, hormonal, and behavioral systems that interact via mutual feedback loops (Thompson et al., 2008).

Furthermore, emotion dysregulation is often conceptualized as the result of interactions between 
"bottom-up" emotional processes and "top-down" control or regulatory processes (Ochsner \& Gross, 2005; Swartz \& Monk, 2014), thus this construct could serve as a useful starting point for integrating findings on the interactions between processes occurring at these levels of analysis. In this section, we provide some examples of how measures of emotion dysregulation have started to be studied in conjunction with neural, psychophysiological, genetic, and epigenetic assessments to begin formulating integrative psychobiological models for the etiology of internalizing symptoms.

Affective neuroscience findings. Recent research has begun probing the neural correlates of emotion regulation and dysregulation (Ochsner \& Gross, 2005). Contemporary theories postulate that deficits in prefrontal control of subcortical regions play a critical role in failure to appropriately contain or terminate emotions (Ahmed, Bittencourt-Hewitt, \& Sebastian, 2015; Davidson, Pizzagalli, Nitschke, \& Putnam, 2002; Macdonald, Goines, Novacek, \& Walker, 2016; Swartz \& Monk, 2014). This is a particularly salient issue in adolescence, when emotional reactivity increases (Dahl \& Gunnar, 2009), but prefrontally-mediated emotion regulation skills are slower to develop (Casey et al., 2010). This disparity is thought to contribute to the rise in psychopathology during this life stage (Ahmed et al., 2015). Supporting theories on the role of prefrontal-limbic interactions in emotion regulation/dysregulation, a recent metaanalysis of functional MRI studies of healthy adults engaged in emotion regulation found a relatively consistent set of neural regions involved (Frank et al., 2014). Across 47 studies of emotion downregulation and 12 studies of emotion upregulation, cortical regions including the superior frontal gyrus, cingulate, and premotor areas showed enhanced activity across all regulation conditions, whereas the amygdala was associated with emotional intensity, revealing 
heightened activity during upregulated states and lower activity in downregulated states (Frank et al., 2014).

Results consistent with the role of the prefrontal cortex in the cognitive control of negative affect are also found in clinical samples of adults. A review of neuroimaging studies comparing depressed and non-depressed adults reported an overall pattern of overactive amygdala, overactive anterior cingulate cortex, and underactive dorsolateral prefrontal cortex (dlPFC) in major depressive disorder (Gotlib \& Hamilton, 2008). This is not surprising, given the role of the amygdala in processing affectively salient stimuli and the role of the dlPFC in cognitive control, which is frequently deficient in depressed patients. A similar pattern emerges for anxiety disorders. For instance, one fMRI study (Ball, Ramsawh, Campbell-Sills, Paulus, \& Stein, 2013) found that adults with Generalized Anxiety Disorder (GAD) and Panic Disorder showed lower activation in dorsolateral prefrontal cortex (dlPFC) and dorsomedial prefrontal cortex (dmPFC) regions during emotion reappraisal and emotion maintenance while viewing negative images compared to healthy controls. These findings suggest insufficient top-down control of negative affect may be risk factors for the development of depressive and anxious symptomatology in adults.

Neuroimaging studies of emotion regulation and dysregulation have begun emerging in children and adolescents as well (for reviews, please see Ahmed et al., 2015; Swartz \& Monk, 2014). Results thus far appear consistent with studies in adults. A recent review of research on the role of amygdala-prefrontal connectivity in emotion regulation concluded that functional and structural alterations in this circuit are related to the development of pediatric anxiety disorders (Swartz \& Monk, 2014), and similar evidence is accruing to support the involvement of this circuit in depressive symptomatology. For instance, 8-12-year-old children who were instructed 
to regulate their responses to negative affect after viewing sad photos exhibit greater activation in prefrontal regions (vlPFC, dlPFC, and dmPFC) compared to viewing the same photos passively without reappraisal (Belden, Luby, Pagliaccio, \& Barch, 2014). Furthermore, there is evidence of age-related improvements in the recruitment of prefrontal regions for reappraisal from childhood to adolescence and into adulthood (McRae et al., 2012). When studying youth (ages 7-15 years old) with a history of major depressive disorder, they show lower levels of activation in the inferior frontal gyrus during a cognitive reappraisal task compared to their never-depressed counterparts (Belden, Pagliaccio, Murphy, Luby, \& Barch, 2015). This pattern of neural activity may constitute a diathesis for the development of depression or a scar of their prior depressive episodes.

In addition to showing evidence of weaker activation in frontal regions, youth exposed to adversity or exhibiting signs of internalizing psychopathology also show evidence of elevated activity in limbic regions involved in emotion processing like the amygdala and the hippocampus. For instance, one study of depressed 4-6-year-old preschoolers revealed a rightlateralized pattern of elevated amygdala, thalamus, inferior frontal gyrus, and angular gyrus activity during face processing in this group compared to the unaffected control group, and amygdala activity was correlated with parent-reported deficits in emotion regulation (Gaffrey, Barch, Singer, Shenoy, \& Luby, 2013). Consistent with this result, a study of 60 school-aged children showed that greater responses to sad faces in emotion reactivity neural regions (e.g. amygdala, hippocampus) predicted lower emotion regulation skills and higher levels of depression (Pagliaccio, Luby, Luking, Belden, \& Barch, 2014). When examining samples at-risk for emotion dysregulation and internalizing, adolescents (ages 13-19) with a prior history of child maltreatment tend to exhibit greater activation in the salience network (including the 
amygdala, putamen, and anterior insula) in response to negative versus neutral stimuli when compared to an unexposed control group (McLaughlin, Peverill, Gold, Alves, \& Sheridan, 2015). Furthermore, adults with a history of childhood maltreatment exhibit positive connectivity between the amygdala and the prefrontal cortex during an emotion-matching fMRI task, whereas non-maltreated adults show negative amygdala-PFC connectivity, a pattern that is consistent with more effective emotion regulation (Jedd et al., 2015).

Overall, these findings suggest that children and adolescents experiencing adversity and/or elevated levels of internalizing symptomatology exhibit heightened activity in limbic regions and/or altered cortico-limbic functional and structural connectivity, which correlates with decreased emotion regulation abilities. This body of evidence supports theoretical models featuring cortico-limbic circuitry as a central underlying neurobiological substrate for emotion regulation, and suggests it as a possible target for early identification of risk for psychopathology as well as a marker for treatment efficacy in internalizing disorders.

Psychophysiological findings. Future neuroimaging studies should integrate these results with psychophysiological theories of emotion dysregulation. Abnormalities in the activity of stress-mediating systems like the autonomic nervous system (especially the parasympathetic branch) and the hypothalamic-pituitary-adrenocortical (HPA) axis can not only serve as useful peripheral indices of emotional reactivity to stressors, but also are thought to indicate deficient prefrontal control of emotion (Appelhans \& Luecken, 2006; Beauchaine, 2015a; Thayer \& Lane, 2009). For instance, respiratory sinus arrhythmia (RSA), an index of parasympathetic influence on cardiac activity, has been consistently associated with better emotion regulation and executive function skills (Appelhans \& Luecken, 2006) and it is thought to reflect medial PFC influences on the peripheral nervous system (Beauchaine, 2015b). Conversely, low RSA has been linked to 
a wide range of mental health conditions, including depression and anxiety, but also to attention problems, conduct disorder, and self-injury (for a review, see Beauchaine, 2015a). Importantly, recent research suggests that adolescents' RSA in response to emotion-eliciting stimuli (e.g., film clips) interacts with maternal emotion socialization practices to predict internalizing symptoms (Hastings, Klimes-Dougan, Kendziora, Brand, \& Zahn-Waxler, 2014). This interaction is such that less supportive and more punitive maternal socialization and greater RSA suppression jointly predict greater levels of fear and sadness in youth (Hastings et al., 2014). While these initial findings are promising, more research will be needed to establish connections between peripheral physiology, neural activity, subjective reports of emotions or emotion regulation strategies, and psychopathology.

Genetics. Similarly, research on genetic markers or moderators of risk for internalizing disorders has burgeoned in the past decade, adding another level of analysis to psychobiological models in developmental psychopathology (Cicchetti \& Natsuaki, 2014). Advances in molecular genetics have supported the hypothesis that individual differences in emotional behavior and the ability to regulate one's emotions are impacted by genetic variation, even though the proportion of variance explained by genes alone tends to be small and more often than not gene-byenvironment interactions qualify the associations found (Hariri \& Holmes, 2006). For instance, we discussed that child maltreatment is associated with greater risk for emotion dysregulation and internalizing symptoms, but these associations are moderated by genotype. There is increasing evidence of gene-by-environment $(\mathrm{G} \times \mathrm{E})$ interactions involving the serotonin transporter (5-HTTLPR), monoamine oxidase A, or CRHR1 genes and environmental adversity, rendering some youth more vulnerable to internalizing psychopathology than others (for recent reviews, see Cicchetti, 2013; Jaffee, 2017). 
Recent studies are also beginning to reveal evidence of G x G x E interactions. For example, one large study of 1,096 maltreated and non-maltreated African American children found of evidence of $\mathrm{G} \times \mathrm{G} \times \mathrm{E}$ interactions between 5-HTTLPR and the norepinephrine transporter gene, as well as 5-HTTLPR and the BDNF gene in interactions with maltreatment as predictors of depressive symptomatology (Cicchetti \& Rogosch, 2014). In addition, there was a G x G x E interaction between the BDNF gene, CRHR1 haplotype, and maltreatment in predicting psychopathology (Cicchetti \& Rogosch, 2014).

Adding another layer of complexity to $\mathrm{G} x \mathrm{E}$ interactions, recent research has found evidence of G x E x E interactions. For example, one longitudinal study identified interactions between genetics (CRHR1 and 5-HTTLPR genes) and both early-life (before age 5) and recent chronic stress proximal to age 20 in predicting depressive symptomatology (Starr, Hammen, Conway, Raposa, \& Brennan, 2014). The overall pattern of results suggests that early-life adversity sensitized individuals to the effects of chronic stress on depressive symptoms, but genetic risk moderated this association such that some individuals were more vulnerable to this sensitizing effect than others (Starr et al., 2014).

Another novel line of investigation has implicated inflammatory processes in the pathophysiology of at least some depression subtypes (Raison \& Miller, 2011). In children, one recent study found that variation in inflammatory genetic variants (specifically, interleukin $1 \beta$ gene, IL-1 $\beta$ ) interact with contextual stressors to predict symptoms of major depressive disorder in 3-5-year-old preschoolers, some of whom were exposed to child maltreatment (Ridout et al., 2014). IL-1 $\beta$ is a proinflammatory cytokine whose production can increase after psychosocial stressors, and animal models have demonstrated its role in perturbing stress neurobiology, with implications for depressive-like behaviors (Dantzer, O'Connor, Freund, Johnson, \& Kelley, 
2008). There is increasing recognition that neuro-immune interactions play a role in the development of stress-related psychopathology (Hostinar, Nusslock, \& Miller, 2017). However, more research is needed to examine these processes developmentally and to elucidate the specific neural pathways through which inflammation confers these risks in human studies.

In sum, genetic variation appears to interact with environmental characteristics to predict psychopathology, but more research is needed to reveal the intermediary pathways underlying these associations in humans: from genes, to proteins, to neural function and processing of environmental input, and finally to emotion and behavior.

Epigenetics. In addition to these gene-by-environment interactions, there is growing recognition that epigenetic regulation of genes is another pathway through which the environment can confer risk for emotion dysregulation and psychopathology. Epigenetic changes (e.g., DNA methylation, posttranslational histone modifications, noncoding RNA molecules) are mechanisms that silence or activate gene expression based on environmental input, including social, nutritional, and toxicological input (Champagne, 2016). There is growing evidence, based on animal models, that prenatal and even preconception social adversity experienced by the mother or father can alter gene expression in the offspring (Champagne, 2016; Doherty \& Roth, 2016). This can occur both directly, by affecting the germline, and indirectly by negatively affecting parental behavior towards the offspring such that parents exposed to stressors tend to provide lower-quality caregiving (Champagne, 2016; Meaney \& Szyf, 2005). The early-life social environment of the offspring then continues to mold the epigenetic landscape, often in sex-specific ways (Massart et al., 2016).

Emerging evidence in humans has begun linking epigenetic alterations to child and adolescent psychopathology. For instance, a few studies using salivary DNA specimens and 
conducting genome-wide methylation analyses have shown that children who experience maltreatment show differential methylation of numerous genes involved in mental health, but also in physical health risks (e.g., immune functioning, cancer) compared to non-maltreated children (Cicchetti, Hetzel, Rogosch, Handley, \& Toth, 2016; Yang et al., 2013). Most other epigenetic studies in youth (and adults) have focused on the epigenetic profiles of genes involved in the regulation of the HPA axis, especially the glucocorticoid receptor gene, NR3C1 (Tyrka, Ridout, \& Parade, 2016). A recent meta-analysis of 23 studies conducted with samples of various ages found evidence that prenatal and early-life social adversity are both associated with hypermethylation of $\mathrm{NR} 3 \mathrm{C} 1$, which was correlated with greater cortisol stress responses as well as psychopathology in some studies (Palma-Gudiel, Cordova-Palomera, Leza, \& Fananas, 2015). In youth, higher levels of methylation of NR3C1 in varying biological specimens (saliva, whole blood) has been associated with psychosocial adversity in preschoolers (Tyrka et al., 2015), older children (Cicchetti \& Handley, 2017), and adolescents (Romens, McDonald, Svaren, \& Pollak, 2015; van der Knaap et al., 2014). However, more research is needed to then link NR3C1 methylation to emotion regulation/dysregulation and to psychopathology in youth. There is some emerging evidence that this may be the case. For example, one study comparing maltreated and non-maltreated children found that hypermethylation of NR3C1 was associated with prior experiences of maltreatment and also to greater emotional lability-negativity and greater depressive symptoms among 9-year-old children (Cicchetti \& Handley, 2017).

The findings we summarized here are significant because they begin to build the conceptual and empirical bridges necessary to create multilevel, mechanistic models of how mood and anxiety symptoms develop. Most human research on genetics and epigenetics thus far has focused on "bottom-up" influences on emotion regulation/dysregulation -i.e., influences on 
biological processes involved in stress and emotional reactivity. As a result, we understand little about genetic (or gene-by-environment interactions) and epigenetic influences on cognitive control processes that would attempt to modulate or terminate emotional reactions to adverse stimuli and experiences. We also understand little about how genetic and epigenetic influences on emotional processes may constrain the repertoire and effectiveness of emotion regulation strategies that youth can acquire and deploy. Furthermore, more research is needed to connect findings at the neural, physiological, genetic, epigenetic, and socio-cultural levels of analysis to understand which risk factors for emotion dysregulation are independent from each other versus part of a network of inter-connected alterations in behavior, brain, stress physiology, and genes.

\section{Conclusions, Future Directions, and Implications for Interventions}

In this brief review, we focused on the developmental origins of emotion dysregulation, recent findings on the temporal ordering and potential causal involvement of emotion dysregulation in psychopathology, and some putative biological underpinnings of emotion dysregulation. We concisely summarize our conclusions here.

From a developmental perspective, a well-established body of evidence now suggests that early-life experiences within the family and peer contexts are critical for the development of emotion processing and emotion regulation systems. Adding to this evidence, novel findings from epigenetics also suggest that the effects of social adversity on the neurobiology of emotion and stress may begin prenatally or even preconception and can be transmitted intergenerationally. This suggests that emotion dysregulation and psychopathology may have some of their origins much earlier than previously thought, though later social environments continue to shape developmental trajectories. We also know that environmental enrichment at various developmental stages (from prenatal to adult) can lead to epigenetic changes that 
promote positive long-term behavioral adaptation (Doherty \& Roth, 2016). The implication then is that efforts to promote resilience against psychopathology could begin much earlier in development, with the potential to have positive ramifications for mental and physical health across the lifespan. However, an important gap in the literature is to better characterize the early stages in the development of emotion dysregulation (e.g. prenatal influences and infancy), as most research on emotion dysregulation and psychopathology thus far has been conducted with children, adolescents, and adults.

As summarized above, accumulating evidence also suggests that emotion dysregulation often precedes and may contribute causally to the development of internalizing symptomatology. However, what remains unclear is the extent to which emotion dysregulation as an antecedent factor is independent from social-contextual stressors, or simply a proxy measure of their occurrence. Currently, emotion regulation and dysregulation have been largely studied as individual-level processes, with insufficient attention being dedicated to the role of the current social context in shaping what is adaptive or maladaptive (Aldao, 2013). Greater attention to individual sociodemographic characteristics (age, gender, race/ethnicity), the ecological validity of the emotion stimuli used and to interpersonal interactions has the potential to reveal novel and important dimensions of emotion and emotion dysregulation. For instance, emerging findings on the contagious nature of affect and on the dynamic physiological synchrony of the sympathetic and parasympathetic nervous system in dyads -e.g., mothers and infants (Waters, West, Karnilowicz, \& Mendes, 2017) suggest that we might be missing significant information if we consider emotions as simple responses of the individual to stimuli in the environment. Future research could benefit from contextualizing individual emotion regulation/dysregulation more, by devoting greater attention to the social learning history of the individual and to any current 
interpersonal relationships, their affective tone and their encouragement or discouragement of specific emotion-regulatory strategies.

Finally, as illustrated in this review, interest in the neurobiological, physiological, genetic, and epigenetic processes involved in the development of internalizing disorders has grown exponentially in the past decade. However, what remains unspecified is how exactly emotional stimuli lead to responses -i.e., what are the intermediary links between them and how does the organism determine the sequence of neurocognitive and peripheral physiological changes that are appropriate to deploy for each situation (A. S. Fox, Lapate, Davidson, \& Shackman, in press)? We also understand little about the long-term developmental processes through which momentary instances of emotion dysregulation accumulate and interact with environmental contingencies to create more persistent states, moods, and ultimately disorders associated with clinical impairment that will require treatment or intervention.

What future waves of research also need to reveal are the many missing links between indices of neural, physiological, genetic, and epigenetic activity and internalizing symptoms. The constructs of emotion, emotion regulation, and emotion dysregulation may prove fruitful as starting points in this search for psychobiological mediators and possible targets for intervention, as these constructs are proving themselves to be a useful and intuitive interface between the biological and psychological levels of analysis, as highlighted in various studied we discussed here. Additionally, integrative conceptual and computational models that synthesize the wealth of information gleaned from such multilevel analyses need to be developed to improve theoretical models of internalizing spectrum disorders, as well as the prediction and treatment of mental illness. 
As far as implications for preventative interventions are concerned, it is evident that reducing the incidence of child maltreatment and peer victimization, as well as training parents to model healthier emotion regulation strategies for their own benefit and for that of their children would likely result in reductions in the prevalence of internalizing symptomatology. Furthermore, multilevel models in developmental psychopathology suggest that resilience can be sparked at any level (neural, physiological, behavioral, etc.), but environmental supports need to be in place to create and maintain positive synergies within children and adolescents that will result in optimal mental and physical health outcomes across the lifespan. 


\section{References}

Adrian, M., Zeman, J., \& Veits, G. (2011). Methodological implications of the affect revolution: A 35-year review of emotion regulation assessment in children. Journal of Experimental Child Psychology, 110(2), 171-197. doi:10.1016/j.jecp.2011.03.009

Ahmed, S. P., Bittencourt-Hewitt, A., \& Sebastian, C. L. (2015). Neurocognitive bases of emotion regulation development in adolescence. Developmental Cognitive Neuroscience, 15, 11-25. doi:10.1016/j.den.2015.07.006

Ainsworth, M. D. S., Bell, S. M., \& Stayton, D. J. (1974). Infant-mother attachment and social development: "Socialisation" as a product of reciprocal responsiveness to signals. In M. P. Richards (Ed.), The integration of the child into a social world (pp. 99-135). London: Cambridge University Press.

Aldao, A. (2013). The future of emotion regulation research: Capturing context. Perspectives on Psychological Science, 8(2), 155-172. doi:10.1177/1745691612459518

Aldao, A., Nolen-Hoeksema, S., \& Schweizer, S. (2010). Emotion-regulation strategies across psychopathology: A meta-analytic review. Clinical Psychology Review, 30(2), 217-237. doi:10.1016/j.cpr.2009.11.004

Appelhans, B. M., \& Luecken, L. J. (2006). Heart rate variability as an index of regulated emotional responding. Review of General Psychology, 10(3), 229-240. doi:10.1037/10892680.10.3.229

Ball, T. M., Ramsawh, H. J., Campbell-Sills, L., Paulus, M. P., \& Stein, M. B. (2013). Prefrontal dysfunction during emotion regulation in generalized anxiety and panic disorders. Psychological Medicine, 43(7), 1475-1486. doi:10.1017/S0033291712002383 
Banny, A. M., Cicchetti, D., Rogosch, F. A., Oshri, A., \& Crick, N. R. (2013). Vulnerability to depression: A moderated mediation model of the roles of child maltreatment, peer victimization, and serotonin transporter linked polymorphic region genetic variation among children from low socioeconomic status backgrounds. Development and Psychopathology, 25(3), 599-614. doi:10.1017/S0954579413000047

Beauchaine, T. P. (2015a). Future directions in emotion dysregulation and youth psychopathology. Journal of Clinical Child and Adolescent Psychology, 44(5), 875-896. doi:10.1080/15374416.2015.1038827

Beauchaine, T. P. (2015b). Respiratory sinus arrhythmia: A transdiagnostic biomarker of emotion dysregulation and psychopathology. Current Opinion in Psychology, 3, 43-47. doi:10.1016/j.copsyc.2015.01.017

Beauchaine, T. P., \& Gatzke-Kopp, L. M. (2012). Instantiating the multiple levels of analysis perspective in a program of study on externalizing behavior. Development and Psychopathology, 24(3), 1003-1018. doi: 10.1017/S0954579412000508

Behar, E., DiMarco, I. D., Hekler, E. B., Mohlman, J., \& Staples, A. M. (2009). Current theoretical models of generalized anxiety disorder (GAD): Conceptual review and treatment implications. Journal of Anxiety Disorders, 23(8), 1011-1023. doi:10.1016/j.janxdis.2009.07.006

Belden, A. C., Luby, J. L., Pagliaccio, D., \& Barch, D. M. (2014). Neural activation associated with the cognitive emotion regulation of sadness in healthy children. Developmental Cognitive Neuroscience, 9, 136-147. doi:10.1016/j.den.2014.02.003 
Belden, A. C., Pagliaccio, D., Murphy, E. R., Luby, J. L., \& Barch, D. M. (2015). Neural activation during cognitive emotion regulation in previously depressed compared to healthy children: Evidence of specific alterations. Journal of the American Academy of Child and Adolescent Psychiatry, 54(9), 771-781. doi:10.1016/j.jaac.2015.06.014

Bosquet, M., \& Egeland, B. (2006). The development and maintenance of anxiety symptoms from infancy through adolescence in a longitudinal sample. Development and Psychopathology, 18, 517-550. doi:10.10170S0954579406060275

Bradley, B., DeFife, J. A., Guarnaccia, C., Phifer, J., Fani, N., Ressler, K. J., \& Westen, D. (2011). Emotion dysregulation and negative affect: Association with psychiatric symptoms. Journal of Clinical Psychiatry, 72(5), 685-691. doi:10.4088/JCP.10m06409b1u

Braithwaite, E. C., O'Connor, R. M., Degli-Esposti, M., Luke, N., \& Bowes, L. (2017). Modifiable predictors of depression following childhood maltreatment: A systematic review and meta-analysis. Translational Psychiatry, 7(7), e1162. doi:10.1038/tp.2017.140

Brenning, K. M., Soenens, B., Braet, C., \& Bosmans, G. (2012). Attachment and depressive symptoms in middle childhood and early adolescence: Testing the validity of the emotion regulation model of attachment. Personal Relationships, 19(3), 445-464.

Brumariu, L. E., \& Kerns, K. A. (2010). Parent-child attachment and internalizing symptoms in childhood and adolescence: A review of empirical findings and future directions. Development and Psychopathology, 22(1), 177-203. doi:10.1017/S0954579409990344 
Calkins, S., \& Hill, A. (2007). Caregiver influences on emerging emotion regulation: Biological and environmental transactions in early development. In J. J. Gross (Ed.), Handbook of Emotion Regulation (pp. 229-248). New York, NY: Guilford Press.

Casey, B. J., Jones, R. M., Levita, L., Libby, V., Pattwell, S. S., Ruberry, E. J., . . Somerville, L. H. (2010). The storm and stress of adolescence: Insights from human imaging and mouse genetics. Developmental Psychobiology, 52(3), 225-235. doi:10.1002/dev.20447

Cassidy, J. (1994). Emotion regulation: Influences of attachment relationships. Monographs of the Society for Research in Child Development, 59(2-3), 228-249.

Champagne, F. A. (2016). Epigenetic legacy of parental experiences: Dynamic and interactive pathways to inheritance. Development and Psychopathology, 28(4), 1219-1228. doi:10.1017/S0954579416000808

Ciarrochi, J., Heaven, P. C. L., \& Supavadeeprasit, S. (2008). The link between emotion identification skills and socio-emotional functioning in early adolescence: A 1-year longitudinal study. Journal of Adolescence, 31(5), 565-582. doi:10.1016/j.adolescence.2007.10.004

Cicchetti, D. (1984). The emergence of developmental psychopathology. Child Development, 55(1), 1-7. doi: 10.2307/1129830

Cicchetti, D. (2013). Annual Research Review: Resilient functioning in maltreated children past, present, and future perspectives. Journal of Child Psychology and Psychiatry, 54(4), 402-422. doi:10.1111/j.1469-7610.2012.02608.x

Cicchetti, D. (2016). Socioemotional, personality, and biological development: Illustrations from a multilevel developmental psychopathology perspective on child maltreatment. Annual Review of Psychology, 67, 187-211. doi:10.1146/annurev-psych-122414-033259 
Cicchetti, D., Ackerman, B. P., \& Izard, C. E. (1995). Emotions and emotion regulation in developmental psychopathology. Development and Psychopathology, 7(1), 1-10.

Cicchetti, D., \& Handley, E. D. (2017). Methylation of the glucocorticoid receptor gene (NR3C1) in maltreated and nonmaltreated children: Associations with behavioral undercontrol, emotional lability/negativity, and externalizing and internalizing symptoms. Development and Psychopathology, 29(5), 1795-1806.

Cicchetti, D., Hetzel, S., Rogosch, F. A., Handley, E. D., \& Toth, S. L. (2016). An investigation of child maltreatment and epigenetic mechanisms of mental and physical health risk. Development and Psychopathology, 28(4), 1305-1317. doi:10.1017/S0954579416000869

Cicchetti, D., \& Natsuaki, M. N. (2014). Multilevel developmental perspectives toward understanding internalizing psychopathology: Current research and future directions. Development and Psychopathology, 26(4), 1189-1190. doi:10.1017/S0954579414000959

Cicchetti, D., \& Ng, R. (2014). Emotional development in maltreated children. Children and Emotion: New Insights into Developmental Affective Science, 26, 29-41. doi:10.1159/000354349

Cicchetti, D., \& Rogosch, F. A. (2014). Genetic moderation of child maltreatment effects on depression and internalizing symptoms by serotonin transporter linked polymorphic region (5-HTTLPR), brain-derived neurotrophic factor (BDNF), norepinephrine transporter (NET), and corticotropin releasing hormone receptor 1 (CRHR1) genes in African American children. Development and Psychopathology, 26(4), 1219-1239. doi:10.1017/S0954579414000984 
Cicchetti, D., \& Toth, S. L. (2005). Child maltreatment. Annual Review of Clinical Psychology, 1, 409-438. doi:10.1146/annurev.clinpsy.1.102803.144029

Cole, P. M., \& Hall, S. E. (2010). Emotion dysregulation as a risk factor for psychopathology. In T. Beauchaine \& S. Hinshaw (Eds.), Child and Adolescent Psychopathology (pp. 265300). Hoboken, N.J. : John Wiley \& Sons.

Cole, P. M., Martin, S. E., \& Dennis, T. A. (2004). Emotion regulation as a scientific construct: Methodological challenges and directions for child development research. Child Development, 75(2), 317-333. doi:DOI 10.1111/j.1467-8624.2004.00673.x

Dahl, R. E., \& Gunnar, M. R. (2009). Heightened stress responsiveness and emotional reactivity during pubertal maturation: Implications for psychopathology. Development and Psychopathology, 21(1), 1-6. doi:10.1017/S0954579409000017

Dantzer, R., O'Connor, J. C., Freund, G. G., Johnson, R. W., \& Kelley, K. W. (2008). From inflammation to sickness and depression: When the immune system subjugates the brain. Nature Reviews Neuroscience, 9(1), 46-56. doi:10.1038/nrn2297

Davidson, R. J., Pizzagalli, D., Nitschke, J. B., \& Putnam, K. (2002). Depression: Perspectives from affective neuroscience. Annual Review of Psychology, 53, 545-574. doi:DOI 10.1146/annurev.psych.53.100901.135148

Doherty, T. S., \& Roth, T. L. (2016). Insight from animal models of environmentally driven epigenetic changes in the developing and adult brain. Development and Psychopathology, 28(4), 1229-1243. doi:10.1017/S095457941600081x

Dougherty, L. R., Klein, D. N., Rose, S., \& Laptook, R. S. (2011). Hypothalamic-pituitaryadrenal axis reactivity in the preschool-age offspring of depressed parents: Moderation by early parenting. Psychological Science, 22(5), 650-658. doi:10.1177/0956797611404084 
Eisenberg, N., Cumberland, A., \& Spinrad, T. L. (1998). Parental socialization of emotion. Psychological Inquiry, 9(4), 241-273. doi: 10.1207/s15327965pli0904_1

Eyden, J., Winsper, C., Wolke, D., Broome, M. R., \& MacCallum, F. (2016). A systematic review of the parenting and outcomes experienced by offspring of mothers with borderline personality pathology: Potential mechanisms and clinical implications. Clinical Psychology Review, 47, 85-105. doi:10.1016/j.cpr.2016.04.002

Feng, X., Keenan, K., Hipwell, A. E., Henneberger, A. K., Rischall, M. S., Butch, J., .. . Babinski, D. E. (2009). Longitudinal associations between emotion regulation and depression in preadolescent girls: Moderation by the caregiving environment. Developmental Psychology, 45(3), 798-808. doi:10.1037/a0014617

Fox, A. S., Lapate, R. C., Davidson, R. J., \& Shackman, A. J. (in press). Epilogue-The nature of emotion: A research agenda for the 21 st century. . In A. S. Fox, R. C. Lapate, A. J. Shackman, \& R. J. Davidson (Eds.), The nature of emotion: Fundamental questions (2nd ed.). New York: Oxford University Press.

Fox, N. A. (1994). The development of emotion regulation - Biological and behavioral considerations. Monographs of the Society for Research in Child Development, 59(2-3).

Frank, D. W., Dewitt, M., Hudgens-Haney, M., Schaeffer, D. J., Ball, B. H., Schwarz, N. F., . . . Sabatinelli, D. (2014). Emotion regulation: Quantitative meta-analysis of functional activation and deactivation. Neuroscience and Biobehavioral Reviews, 45, 202-211. doi:10.1016/j.neubiorev.2014.06.010 
Gaffrey, M. S., Barch, D. M., Singer, J., Shenoy, R., \& Luby, J. L. (2013). Disrupted amygdala reactivity in depressed 4-to 6-year-old children. Journal of the American Academy of Child and Adolescent Psychiatry, 52(7), 737-746. doi:10.1016/j.jaac.2013.04.009

Goldin, P. R., McRae, K., Ramel, W., \& Gross, J. J. (2008). The neural bases of emotion regulation: Reappraisal and suppression of negative emotion. Biological Psychiatry, 63(6), 577-586. doi:10.1016/j.biopsych.2007.05.031

Gotlib, I. H., \& Hamilton, J. P. (2008). Neuroimaging and depression: Current status and unresolved issues. Current Directions in Psychological Science, 17(2), 159-163. doi:DOI 10.1111/j.1467-8721.2008.00567.x

Gotlib, I. H., \& Joormann, J. (2010). Cognition and depression: Current status and future directions. Annual Review of Clinical Psychology, 6, 285-312. doi:10.1146/annurev.clinpsy.121208.131305

Gross, J. J. (2013). Emotion regulation: Taking stock and moving forward. Emotion, 13(3), 359365. doi:10.1037/a0032135

Guyer, A. E., Kaufman, J., Hodgdon, H. B., Masten, C. L., Jazbec, S., Pine, D. S., \& Ernst, M. (2006). Behavioral alterations in reward system function: The role of childhood maltreatment and psychopathology. Journal of the American Academy of Child and Adolescent Psychiatry, 45(9), 1059-1067. doi:10.1097/01.chi.0000227882.50404.11

Hariri, A. R., \& Holmes, A. (2006). Genetics of emotional regulation: The role of the serotonin transporter in neural function. Trends in Cognitive Sciences, 10(4), 182-191. doi:10.1016/j.tics.2006.02.011

Hastings, P. D., Klimes-Dougan, B., Kendziora, K. T., Brand, A., \& Zahn-Waxler, C. (2014). Regulating sadness and fear from outside and within: Mothers' emotion socialization and 
adolescents' parasympathetic regulation predict the development of internalizing difficulties. Development and Psychopathology, 26(4), 1369-1384. doi:10.1017/S0954579414001084

Hatzenbuehler, M. L., McLaughlin, K. A., \& Nolen-Hoeksema, S. (2008). Emotion regulation and internalizing symptoms in a longitudinal study of sexual minority and heterosexual adolescents. Journal of Child Psychology and Psychiatry, 49(12), 1270-1278. doi:10.1111/j.1469-7610.2008.01924.x

Hostinar, C. E., Nusslock, R., \& Miller, G. E. (2017). Future directions in the study of early-life stress and physical and emotional health: Implications of the neuroimmune network hypothesis. Journal of Clinical Child and Adolescent Psychology, 1-15. doi:10.1080/15374416.2016.1266647

Insel, T., Cuthbert, B., Garvey, M., Heinssen, R., Pine, D. S., Quinn, K., . . Wang, P. (2010). Research Domain Criteria (RDoC): Toward a new classification framework for research on mental disorders. American Journal of Psychiatry, 167(7), 748-751. doi:10.1176/appi.ajp.2010.09091379

Jaffee, S. R. (2017). Child maltreatment and risk for psychopathology in childhood and adulthood. Annual Review of Clinical Psychology, 13, 525-551. doi:10.1146/annurevclinpsy-032816-045005

Jedd, K., Hunt, R. H., Cicchetti, D., Hunt, E., Cowell, R. A., Rogosch, F. A., . . Thomas, K. M. (2015). Long-term consequences of childhood maltreatment: Altered amygdala functional connectivity. Development and Psychopathology, 27(4), 1577-1589. doi:10.1017/S0954579415000954 
Kim, J., \& Cicchetti, D. (2010). Longitudinal pathways linking child maltreatment, emotion regulation, peer relations, and psychopathology. Journal of Child Psychology and Psychiatry, 51(6), 706-716. doi:10.1111/j.1469-7610.2009.02202.x

Kim-Spoon, J., Cicchetti, D., \& Rogosch, F. A. (2013). A longitudinal study of emotion regulation, emotion lability-negativity, and internalizing symptomatology in maltreated and nonmaltreated children. Child Development, 84(2), 512-527. doi:10.1111/j.14678624.2012.01857.x

Li, M., D'Arcy, C., \& Meng, X. (2016). Maltreatment in childhood substantially increases the risk of adult depression and anxiety in prospective cohort studies: Systematic review, meta-analysis, and proportional attributable fractions. Psychological Medicine, 46(4), 717-730. doi:10.1017/S0033291715002743

Luke, N., \& Banerjee, R. (2013). Differentiated associations between childhood maltreatment experiences and social understanding: A meta-analysis and systematic review. Developmental Review, 33(1), 1-28. doi:10.1016/j.dr.2012.10.001

Macdonald, A. N., Goines, K. B., Novacek, D. M., \& Walker, E. F. (2016). Prefrontal mechanisms of comorbidity from a transdiagnostic and ontogenic perspective. Development and Psychopathology, 28(4), 1147-1175. doi:10.1017/S0954579416000742

Main, M., \& Solomon, J. (1986). Discovery of a new, insecure-disorganized/disoriented attachment pattern. In T. B. Brazelton \& M. Yogman (Eds.), Affective development in infancy (pp. 95-124). Norwood, New Jersey: Ablex.

Massart, R., Nemoda, Z., Suderman, M. J., Sutti, S., Ruggiero, A. M., Dettmer, A. M., . . Szyf, M. (2016). Early life adversity alters normal sex-dependent developmental dynamics of 
DNA methylation. Development and Psychopathology, 28(4pt2), 1259-1272. doi:10.1017/S0954579416000833

Maughan, A., \& Cicchetti, D. (2002). Impact of child maltreatment and interadult violence on children's emotion regulation abilities and socioemotional adjustment. Child Development, 73(5), 1525-1542. doi:Doi 10.1111/1467-8624.00488

Maughan, A., Cicchetti, D., Toth, S. L., \& Rogosch, F. A. (2007). Early-occurring maternal depression and maternal negativity in predicting young children's emotion regulation and socioemotional difficulties. Journal of Abnormal Child Psychology, 35(5), 685-703. doi:10.1007/s10802-007-9129-0

McLaughlin, K. A., Hatzenbuehler, M. L., \& Hilt, L. M. (2009). Emotion dysregulation as a mechanism linking peer victimization to internalizing symptoms in adolescents. Journal of Consulting and Clinical Psychology, 77(5), 894-904. doi:10.1037/a0015760

McLaughlin, K. A., Hatzenbuehler, M. L., Mennin, D. S., \& Nolen-Hoeksema, S. (2011). Emotion dysregulation and adolescent psychopathology: A prospective study. Behaviour Research and Therapy, 49(9), 544-554. doi:10.1016/j.brat.2011.06.003

McLaughlin, K. A., Peverill, M., Gold, A. L., Alves, S., \& Sheridan, M. A. (2015). Child maltreatment and neural systems underlying emotion regulation. Journal of the American Academy of Child and Adolescent Psychiatry, 54(9), 753-762.

doi:10.1016/j.jaac.2015.06.010

McRae, K., Gross, J. J., Weber, J., Robertson, E. R., Sokol-Hessner, P., Ray, R. D., . . Ochsner, K. N. (2012). The development of emotion regulation: An fMRI study of cognitive reappraisal in children, adolescents and young adults. Social Cognitive and Affective Neuroscience, 7(1), 11-22. doi:10.1093/scan/nsr093 
Meaney, M. J., \& Szyf, M. (2005). Environmental programming of stress responses through DNA methylation: life at the interface between a dynamic environment and a fixed genome. Dialogues Clin Neurosci, 7(2), 103-123.

Mennin, D. S., Heimberg, R. G., Turk, C. L., \& Fresco, D. M. (2002). Applying an emotion regulation framework to integrative approaches to generalized anxiety disorder. Clinical Psychology-Science and Practice, 9(1), 85-90. doi:DOI 10.1093/clipsy/9.1.85

Morris, A. S., Silk, J. S., Steinberg, L., Myers, S. S., \& Robinson, L. R. (2007). The role of the family context in the development of emotion regulation. Social Development, 16(2), 361-388. doi:10.1111/j.1467-9507.2007.00389.x

Nanni, V., Uher, R., \& Danese, A. (2012). Childhood maltreatment predicts unfavorable course of illness and treatment outcome in depression: A meta-analysis. Am J Psychiatry, 169(2), 141-151. doi:10.1176/appi.ajp.2011.11020335

Nelson, J., Klumparendt, A., Doebler, P., \& Ehring, T. (2017). Childhood maltreatment and characteristics of adult depression: Meta-analysis. Br J Psychiatry, 210(2), 96-104. doi:10.1192/bjp.bp.115.180752

Nolen-Hoeksema, S., Wisco, B. E., \& Lyubomirsky, S. (2008). Rethinking rumination. Perspectives on Psychological Science, 3(5), 400-424. doi:10.1111/j.17456924.2008.00088.x

Ochsner, K. N., \& Gross, J. J. (2005). The cognitive control of emotion. Trends in Cognitive Sciences, 9(5), 242-249. doi:10.1016/j.tics.2005.03.010

Pagliaccio, D., Luby, J. L., Luking, K. R., Belden, A. C., \& Barch, D. M. (2014). Brain-behavior relationships in the experience and regulation of negative emotion in healthy children: 
Implications for risk for childhood depression. Development and Psychopathology, 26(4), 1289-1303. doi:10.1017/S0954579414001035

Palma-Gudiel, H., Cordova-Palomera, A., Leza, J. C., \& Fananas, L. (2015). Glucocorticoid receptor gene (NR3C1) methylation processes as mediators of early adversity in stressrelated disorders causality: A critical review. Neuroscience and Biobehavioral Reviews, 55, 520-535. doi:10.1016/j.neubiorev.2015.05.016

Pollak, S. D. (2015). Multilevel developmental approaches to understanding the effects of child maltreatment: Recent advances and future challenges. Development and Psychopathology, 27(4), 1387-1397. doi:10.1017/S0954579415000826

Pollak, S. D., Cicchetti, D., Hornung, K., \& Reed, A. (2000). Recognizing emotion in faces: Developmental effects of child abuse and neglect. Developmental Psychology, 36(5), 679-688. doi:10.1037//0012-1649.36.5.679

Raison, C. L., \& Miller, A. H. (2011). Is depression an inflammatory disorder? Curr Psychiatry Rep, 13(6), 467-475. doi:10.1007/s11920-011-0232-0

Regier, D. A., Kuhl, E. A., \& Kupfer, D. J. (2013). The DSM-5: Classification and criteria changes. World Psychiatry, 12(2), 92-98. doi:10.1002/wps.20050

Ridout, K. K., Parade, S. H., Seifer, R., Price, L. H., Gelernter, J., Feliz, P., \& Tyrka, A. R. (2014). Interleukin 1B gene (IL1B) variation and internalizing symptoms in maltreated preschoolers. Development and Psychopathology, 26(4), 1277-1287.

doi:10.1017/S0954579414001023 
Romens, S. E., McDonald, J., Svaren, J., \& Pollak, S. D. (2015). Associations Between Early Life Stress and Gene Methylation in Children. Child Development, 86(1), 303-309. doi:10.1111/cdev.12270

Romens, S. E., \& Pollak, S. D. (2012). Emotion regulation predicts attention bias in maltreated children at-risk for depression. Journal of Child Psychology and Psychiatry, 53(2), 120127. doi:10.1111/j.1469-7610.2011.02474.x

Schafer, J. O., Naumann, E., Holmes, E. A., Tuschen-Caffier, B., \& Samson, A. C. (2017). Emotion regulation strategies in depressive and anxiety symptoms in youth: A metaanalytic review. Journal of Youth and Adolescence, 46(2), 261-276. doi:10.1007/s10964016-0585-0

Shackman, J. E., \& Pollak, S. D. (2014). Impact of physical maltreatment on the regulation of negative affect and aggression. Development and Psychopathology, 26(4), 1021-1033. doi:10.1017/S0954579414000546

Sheppes, G., Suri, G., \& Gross, J. J. (2015). Emotion regulation and psychopathology. Annual Review of Clinical Psychology, Vol 11, 11, 379-405. doi:10.1146/annurev-clinpsy032814-112739

Shields, A., \& Cicchetti, D. (1998). Reactive aggression among maltreated children: The contributions of attention and emotion dysregulation. Journal of Clinical Child Psychology, 27(4), 381-395. doi:DOI 10.1207/s15374424jccp2704_2

Solomon, R. C. (2002). Back to basics: On the very idea of "basic emotions". Journal for the Theory of Social Behaviour, 32(2), 115-144. doi: 10.1111/1468-5914.00180 
Starr, L. R., Hammen, C., Conway, C. C., Raposa, E., \& Brennan, P. A. (2014). Sensitizing effect of early adversity on depressive reactions to later proximal stress: Moderation by polymorphisms in serotonin transporter and corticotropin releasing hormone receptor genes in a 20-year longitudinal study. Development and Psychopathology, 26(4), 12411254. doi:10.1017/S0954579414000996

Swartz, J. R., \& Monk, C. S. (2014). The role of corticolimbic circuitry in the development of anxiety disorders in children and adolescents. Current Topics in Behavioral Neuroscience, 16, 133-148. doi:10.1007/7854_2013_242

Tandon, M., Cardeli, E., \& Luby, J. (2009). Internalizing disorders in early Childhood: A review of depressive and anxiety disorders. Child and Adolescent Psychiatric Clinics of North America, 18(3), 593-+. doi:10.1016/j.chc.2009.03.004

Thayer, J. F., \& Lane, R. D. (2009). Claude Bernard and the heart-brain connection: Further elaboration of a model of neurovisceral integration. Neuroscience and Biobehavioral Reviews, 33(2), 81-88. doi:10.1016/j.neubiorev.2008.08.004

Thompson, R. A., Lewis, M. D., \& Calkins, S. D. (2008). Reassessing emotion regulation. Child Development Perspectives, 2(3), 124-131. doi:DOI 10.1111/j.1750-8606.2008.00054.x

Thompson, R. A., \& Meyer, S. (2007). Socialization of emotion regulation in the family. In J. J. Gross (Ed.), Handbook of Emotion Regulation (pp. 249-268). New York, NY: Guilford Press.

Tyrka, A. R., Parade, S. H., Eslinger, N. M., Marsit, C. J., Lesseur, C., Armstrong, D. A., . . Seifer, R. (2015). Methylation of exons 1D, 1F, and 1H of the glucocorticoid receptor gene promoter and exposure to adversity in preschool-aged children. Development and Psychopathology, 27(2), 577-585. doi:10.1017/S0954579415000176 
Tyrka, A. R., Ridout, K. K., \& Parade, S. H. (2016). Childhood adversity and epigenetic regulation of glucocorticoid signaling genes: Associations in children and adults. Development and Psychopathology, 28(4), 1319-1331. doi:10.1017/S0954579416000870 van der Knaap, L. J., Riese, H., Hudziak, J. J., Verbiest, M. M. P. J., Verhulst, F., Oldehinkel, A. J., \& van Oort, F. V. A. (2014). Glucocorticoid receptor gene (NR3C1) methylation following stressful events between birth and adolescence. The TRAILS study. Translational Psychiatry, 4. doi:ARTN e38110.1038/tp.2014.22

Waters, S. F., West, T. V., Karnilowicz, H. R., \& Mendes, W. B. (2017). Affect contagion between mothers and infants: Examining valence and touch. Journal of Experimental Psychology-General, 146(7), 1043-1051. doi:10.1037/xge0000322

Yang, B. Z., Zhang, H., Ge, W., Weder, N., Douglas-Palumberi, H., Perepletchikova, F., .. . Kaufman, J. (2013). Child abuse and epigenetic mechanisms of disease risk. American Journal of Preventive Medicine, 44(2), 101-107. doi:10.1016/j.amepre.2012.10.012

Yap, M. B. H., Allen, N. B., \& Sheeber, L. (2007). Using an emotion regulation framework to understand the role of temperament and family processes in risk for adolescent depressive disorders. Clinical Child and Family Psychology Review, 10(2), 180-196. doi:10.1007/s10567-006-0014-0

Zeman, J., Klimes-Dougan, B., Cassano, M., \& Adrian, M. (2007). Measurement issues in emotion research with children and adolescents. Clinical Psychology-Science and Practice, 14(4), 377-401. doi:DOI 10.1111/j.1468-2850.2007.00098.x 